\title{
Subject Index to Volume 89
}

\section{$\mathbf{A}$}

AIDS 42, 105, 157, 162, 531, 549, 570, 1195, 1199,1233

Amebiasis 339, 1214

Arsenic 138

\section{B}

Bacterial Infection 151, 265, 339, 1129

Bartonella spp. 986

Bartonellosis 462, 988, 1219

Book Review 1029, 1032, 1235

Borrelia 288, 460

Bunyaviruses 445

Buruli ulcer 498

\section{C}

Cholera 597, 610, 615, 617, 625, 633, 641, $647,654,665,671,682,688,950,1228$

Clinical studies 592

Coxiella 518

Coxiella burnetii 800

Cryptosporidiosis 157,531

Cycticercosis 919

\section{D}

Dengue 93, 99, 198, 385, 482, 804, 1001, 1043, 1058, 1066, 1081

Diagnosis 3, 11, 46, 63, 99, 105, 116, 165, $232,308,380,403,1088,1117,1122$

Diarrheal Diseases 214, 223, 246, 251, 339, $419,884,892$

Dracunculiasis 5

Drug Resistant Gram Negative Bacteria 960, 965

Drug-Resistance 174, 184, 271, 311

\section{$\mathbf{E}$}

Ecology/Natural History 597, 1066

Editorial 3, 197, 1037

Elimination 51

Emerging Diseases 445, 482, 516

Epidemiology 111, 507, 597, 1095

Eradication 5

Errata 608, 816, 1035

Eye complications 162

\section{F}

Fascioliasis 588, 1212

Filariasis 260, 578, 916

Flaviviruses 516

Fleas 453,462

Fungal Diseases 1195

\section{G}

Genetic Epidemiology 123, 188, 1154

Geohelminths 23, 376

Global Health 130, 138, 214, 223, 229, 232 , $238,246,251,260,265,271,281,293$, $385,407,411,419,426,698,709,717$, $869,873,875,1179,1186,1190$
Gnathostomiasis 63

Gongylonema pulchrum 755

\section{H}

Handwashing 1179

Hantavirus 1006, 489

Health Economics 369, 407, 1146

Health Outcomes 419, 592

Helicobacter 398

Hepatitis B 811

Histoplasmosis 937

Hookworm 540

HTLV-1 549

Hydatid Disease 401

Hydatidosis 554

Hygiene 130, 411, 426

\section{I}

Images in Clinical Tropical Medicine 1, 195, 401, 609, 817, 1038

Immunology 169

Insecticidal curtain 93

\section{K}

KAP 229, 265, 419

\section{$\mathbf{L}$}

Leishmaniasis $105,111,195,345,354$, $359,557,564,570,742,750,899$, 906,1146

Leprosy 781, 1038

Leptospirosis 380, 794, 797, 1088, 1095, 1103

Letter 398, 1033, 1225, 1228, 1233

Lymphatic Filariasis 3, 11, 16

\section{M}

Malaria 178, 184, 188, 311, 317, 326, 403, $466,501,520,527,721,724,737,824$, $840,850,857,866,1117,1122,1129$

Medical Education 281

Medical Entomology 758, 766, 775, 924, 928

Medical history 592

Melioidosis 165, 365, 367, 369, 535, 971, 973, 983

Meningitis 265

Modeling 564

Mosquitoes 68, 78, 466, 482, 1154, 1168

\section{$\mathbf{N}$}

Neglected Tropical Diseases 407

Nematode Infection 1186

Neurocysticercosis 374

Nevirapine 162

Norovirus 1019

Nutrition 130, 223

$\mathbf{O}$

Onchocerciasis 51, 293
Pathogenesis 495

Perspective Piece 819.

Perspectives 5, 198, 403, 610, 819, 1040

Plague 784, 788

Program Management 251, 367, 385

Q

Q fever 991

$\mathbf{R}$

Rabies 1040

Rickettial Diseases 308, 453, 1203

Rift Valley fever 495

Rocio Virus 1013

Rotavirus 246

Schistosomiasis $32,42,46$

Sexually Transmitted Diseases 1199

Shewanella 151

Shigellosis 339

Snake Bite 145

\section{$\mathbf{T}$}

Taeniasis 58

Tembusu virus 1023

Ticks 445,460

Toxoplasmosis 1219

Transmission 51

Travel Medicine 58

Tribute 202

Trichostrongylus 376

Trypanosomiasis 116

Tuberculosis 1, 169, 174, 271, 507, 943, 1233

Tungiasis 932

Typhus 119, 123, 301, 1206

V

Vaccine 419, 434, 1058

Vaccinia 1142

Vector Biology 68, 78

Viral Hemmorhagic Fevers 1135

W

Waterborne Infections 238, 411, 1190

West Nile Virus 197, 205, 211, 1168, 996

\section{$\mathbf{Y}$}

Yellow Fever 434, 1225

$\mathbf{Z}$

Zika virus 516

Zoonotic Diseases 518, 1142, 1212 